\title{
Association of CRP Levels as an Inflammatory Marker in Prognosis of COVID-19 Cases
}

\section{Sharanya $K^{1 *}$, Lakshmi K ${ }^{1}$, Vinod K ${ }^{2}$, Chitralekha $\mathbf{S}^{3}$}

\begin{abstract}
'Associate Professor, Department of Microbiology, Sree Balaji Medical College and Hospital (Bharath Institute of Higher Education \& Research), Chennai, Tamil Nadu, India; ${ }^{2}$ Professor, Department of Anaesthesiology, Sree Balaji Medical College and Hospital (Bharath Institute of Higher Education \& Research), Chennai, Tamil Nadu, India; PProfessor \& HOD, Department of Microbiology, Sree Balaji Medical College and Hospital (Bharath Institute of Higher Education \& Research), Chennai, Tamil Nadu, India.
\end{abstract}

\section{ABSTRACT}

Introduction: The pandemic COVID-19 is an important global threat because of its high infectivity and case fatality rate in highrisk patients. The studies related to physiological mechanisms, pathogenesis, effective laboratory diagnostic methods are still in investigative stages. Several studies on COVID-19 report that inflammatory reactions play a vital role in disease progression.

Objective: In this study, we evaluated the association between the C-Reactive Protein (CRP) and the severity of COVID-19 pneumonia. This study would help the clinicians to monitor the prognosis and severity of the disease.

Methods: A Retrospective study conducted in the Department of Microbiology over 3 months with 336 laboratories confirmed Corona positive patients. Blood samples were collected from these patients and CRP was estimated by the Latex Agglutination test.

Results: Of the 336 patients, 142 (42.2) showed positive results for CRP. Among CRP positive cases, 67 showed high CRP(>24mg/L) and 75 showed low CRP $(\leq 24 \mathrm{mg} / \mathrm{L})$. High CRP was found to be associated with increased severity of the illness.

Conclusion: CRP may be a marker of disease severity and may be a valuable indicator for determining the severity of patients with COVID-19. Continuous CRP monitoring may also predict the prognosis of cases. Further investigations are required to demonstrate the mechanisms by which increased CRP is seen in patients suffering from SARS- CoV-2.

Key Words: CRP, Covid-19, Inflammatory marker, Corona Virus, Pandemic

\section{INTRODUCTION}

Coronavirus belongs to the family Coronaviridae and order Nidoviridales. An unknown viral outbreak has been reported in December 2019, which was later diagnosed to be SARS - CoV2. The pandemic COVID-19 is an important global threat because of its higher infectivity and case fatality rate in high-risk patients. ${ }^{.}$The studies related to physiological mechanisms, pathogenesis, effective laboratory diagnostic methods are still in investigative stages. Appropriate monitoring and proper follow up of treatment strategies are important in the clinical improvement of the cases. Computerized Tomography (CT) scan plays an important role in the assessment of the disease. However, some patients do not show any hypoxemia or respiratory distress which further indicates that the disease is multifaceted.

Several studies on COVID-19 report that inflammatory reactions play a vital role in disease progression. ${ }^{2,3}$ Several in- flammatory markers have been studied to detect the severity and fatality of COVID-19. ${ }^{4}$ Some of the inflammatory markers such as Procalcitonin, serum ferritin, $\mathrm{C}$ reactive protein (CRP), Erythrocyte Sedimentation Rate (ESR) are associated with the severity of COVID-19 disease. ${ }^{5,6}$ Many studies in the recent past have reported that CRP is positively associated with dengue infection indicating that CRP monitoring is one of the reliable biomarkers in predicting the severity of viral infections. ${ }^{7,8}$

We assume that CRP can be used in monitoring the prognosis of the COVID-19 patients. CRP levels help in the early diagnosis of the cases. Patients with severe infection are often associated with high levels of CRP. In this study, we evaluated the association between the CRP and the severity of COVID-19 pneumonia. This study would help the clinicians to monitor the prognosis and severity of the disease.

\section{Corresponding Author:}

Dr. Sharanya Krishnakumar, Associate Professor of Microbiology, Sree Balaji Medical College and Hospital (Bharath Institute of Higher education \& Research),Chennai, India; Phone: 9443166001; Fax: +91-44-2241 6676; Email: sharanyavinod87@gmail.com

ISSN: 2231-2196 (Print)

Received: 05.03 .2021
ISSN: 0975-5241 (Online)

Revised: 12.04 .2021
Accepted: 06.05 .2021
Published: 11.06 .2021 


\section{MATERIALS AND METHODS}

\section{Study design and participants}

This is a retrospective study of 336 adult patients (males and females) admitted with laboratory-confirmed COVID-19. The study was conducted in the Department of Microbiology, Sree Balaji Medical College and Hospital (Bharath Institute of Higher education \& Research), Chennai, Tamil Nadu, India over 3 months (June 2020 to August 2020). Institutional ethical committee approval was obtained.

\section{Procedure}

On admission, the patients with complaints of cough, chest pain and other respiratory or digestive symptoms with or without fever were screened by RT PCR for SARS- CoV2. Only those who were RT PCR positive for SARS-CoV-2 were included in the study. 2-3ml of Venous blood samples were collected from all these patients in a sterile tube without anticoagulant for estimation of CRP. The blood was allowed to clot at room temperature. Serum was separated after centrifugation at $3500 \mathrm{rpm}$ for $10 \mathrm{~min}$. The test was performed immediately using the Qualitative and Semi-Quantitative Rapid Latex slide agglutination test.

\section{Qualitative slide test}

The test serum was placed within the circled area on the special slide provided in the kit to which a drop of CRP latex agent was added. Both were mixed well and the slide was gently rocked for 2 minutes and noted for macroscopic agglutination.

\section{Interpretation}

Coarse agglutination - Strongly positive

Finer agglutination - Weakly positive

Smooth suspension without any noticeable change - Negative

\section{Semi-Quantitative Slide test}

A series of dilutions of a test serum in normal saline (eg:1:2,1:4,1:8 etc) was prepared. One drop of CRP latex reagent was added to them and observed for agglutination for 2 minutes. The highest dilution which shows agglutination is taken as the CRP titre of the test serum.

\section{Interpretation}

The highest dilution that gives agglutination was multiplied with a factor of 6 (sensitivity of antigen 6 microgram $/ \mathrm{ml}$ )

Once CRP was positive, they were categorized into High $(>\mathbf{2 4} \mathbf{m g} / \mathbf{L})$ and low CRP $(<=\mathbf{2 4} \mathbf{m g} / \mathbf{L})$. The patients were followed up until discharge or until they were shifted to higher centres due to deterioration of vitals.

\section{RESULTS}

Of these 67 high positive CRP patients around 65 cases eventually improved with treatment, only 2 cases were shifted to higher centres due to a decrease in saturation (Table 1). All 75 Low positive CRP patients improved well with treatment and got discharged (Table 2).

\section{Table 1: Tabulation of CRP positive cases}

\begin{tabular}{lcc} 
Month & $\begin{array}{c}\text { Total no of Covid posi- } \\
\text { tive cases tested for CRP }\end{array}$ & $\begin{array}{c}\text { Total no of cases } \\
\text { positive for CRP }\end{array}$ \\
\hline June 2020 & 106 & 39 \\
July 2020 & 133 & 53 \\
August 2020 & 97 & 50 \\
Total & 336 & $142(42.2 \%)$ \\
\hline
\end{tabular}

\begin{tabular}{|c|c|c|}
\hline Month & $\begin{array}{l}\text { Total no of cases } \\
\text { with low positive for } \\
\text { CRP }(<=24 \mathrm{mg} / \mathrm{L})\end{array}$ & $\begin{array}{l}\text { Total no of cases } \\
\text { with high positive } \\
\text { for CRP (>24mg/L) }\end{array}$ \\
\hline June 2020 & 25 & 14 \\
\hline July 2020 & 25 & 28 \\
\hline August 2020 & 25 & 25 \\
\hline Total & $75(22.3 \%)$ & $67(19.9 \%)$ \\
\hline
\end{tabular}

\section{DISCUSSION}

The number of patients with COVID-19 is increasing globally with increasing case fatality. Early monitoring of key indicators was an important basis to guide treatment strategies, and early assessment of the severity of the patient condition was of great value. ${ }^{9}$ Level of CRP correlated well with the level of inflammation. Factors such as age, sex, and physical condition do not affect the concentration of CRP. ${ }^{10}$ The production of C-reactive protein activates complement and increases phagocytosis. Hence it reduces the pathogenic microorganisms that attack the body. The level of C-reactive protein is already used for the early diagnosis of pneumonia. ${ }^{I I}$ Patients with severe pneumonia had high CRP levels. It is considered a significant marker in the diagnosis and assessment of severe pulmonary infections. ${ }^{12}$

Our study shows that increasing CRP levels are positively correlating with the progression of the disease. The low positive cases seemed to have a better prognosis than High CRP positive cases. Among the 67 high CRP positive cases, almost 50 per cent of the cases experienced prolonged hospital stay when compared to the low positives. Two of the high positives were shifted to higher centres due to worsening of symptoms. Our study was comparable to a study conducted by Wang et al. which showed that CRP levels increased with the disease progression. ${ }^{9}$ Hence early stage inclusion of CRP levels could reflect on lung lesions and disease prognosis. ${ }^{13}$ 
Another study conducted by Matsumoto et al. also showed similar findings. ${ }^{13}$

Studies show an increase in CRP levels even before critical CT findings are visualized in COVID-19 patients. Hence it acts as an early predictor of severity. Some studies reported other inflammatory markers like Erythrocyte sedimentation rate to be positively associated with CT severity scores. Immunological marker like IL-6 is also found to be increased in non-survivors than survivors. Another important inflammatory marker like increased Procalcitonin also shows a fivefold higher risk of severe SARS CoV2 infection in few studies but it may not be considered as a prognostic marker as it may be influenced by preexisting comorbid conditions. ${ }^{14}$

Another study by Cheng B et al. showed higher neutrophil count: lymphocyte count ratio (NLR) and CRP at admission were good predictors for critical condition and death. ${ }^{15} \mathrm{~A}$ study by Qilin Li et al. suggests a combination of eosinopenia $(<0.02$ $\left.10^{9} / \mathrm{L}\right)$ and elevated hs-CRP $(>4 \mathrm{mg} / \mathrm{L})$ can help in effectively triaging COVID-19 cases from other respiratory diseases in a centre with limited resources. ${ }^{16}$ Another study conducted by Weichenet al. also correlated with our study findings. ${ }^{17}$

Other biomarkers such as high ferritin levels have been described as a predictor of acute respiratory distress syndrome. ${ }^{18}$ Recent studies show decreased lymphocyte counts and increased D-dimer concentrations point out a negative prognosis. Several other biomarkers such as decreased albumin levels, elevated creatine kinase levels and higher LDH levels were also associated with poor prognosis. ${ }^{19}$

\section{CONCLUSION}

Our study shows that CRP may be a marker of disease severity and may be a valuable indicator for determining the severity of patients with COVID-19. Continuous CRP monitoring may also predict the prognosis of cases. Further investigations are required to demonstrate the mechanisms by which increased CRP is seen in patients suffering from SARS- CoV-2.

\section{Conflict of Interest: Nil}

\section{Funding: Nil}

CONTRIBUTION: Study concept, Data collection, Manuscript writing, Final review and approval- Dr Sharanya K. Manuscript writing, Manuscript editing and review: Dr.Lakshmi K, Dr.Vinod K,

Study design and final approval: Dr.ChitralekhaSaikumar.

\section{REFERENCES}

1. Chen N, Zhou M, Deng X, Qu J, GongF, Han Y, et al. Epidemiological and clinical characteristics of 99 cases of 2019 novel coronavirus pneumonia in Wuhan. China: a descriptive study. Lancet. 2020; 10223(395): 507-513.

2. Mehta P, McAuley DF, Browne D, Sanchez E, Tattersall RS, Manson JJ, et al. COVID-19: consider cytokine storm syndromes and immunosuppression. Lancet. 2020;10223(395):1033-1034.

3. Stebbing J, Phelan A, Griffin I, Tucker C, OechsleO, Smith D, et al. COVID-19: combining antiviral and anti-inflammatory treatments. Lancet Infect Dis. 2020;20(4):400-402.

4. Wu C, Chen X, Cai Y, Xia J, Zhou X, Xu S. Risk factors associated with acute respiratory distress syndrome and death in patients with coronavirus disease 2019 pneumonia in Wuhan, China. JAMA Intern Med. 2020;180(7):934-943.

5. Cheng K, Wei M, Shen H, Wu C, Chen D, Xiong W, et al. Clinical characteristics of 463 patients with common and severe type coronavirus disease 2019. Shanghai Med J. 2020;1-15.

6. Gao Y, Li T, Han M, Li X, WuD, Xu Y, et al. Diagnostic utility of clinical laboratory data determinations for patients with the severe COVID-19. J Med Virol. 2020;92(7):791-796.

7. Chen CC, Lee IK, Liu JW, Huang SY, Wang L. Utility of Creactive protein levels for early prediction of dengue severity in adults. Biomed Res Int. 2015;2015:936062.

8. Eppy E, Suhendro S, Nainggolan L, Rumende CM. The differences between interleukin- 6 and c-reactive protein levels among adult patients of dengue infection with and without plasma leakage. Acta Med Indones. 2016;48(1):3-9.

9. Wang L. C-reactive protein levels in the early stage of COVID-19. Med Mal Infect. 2020;50(4):332-334.

10. Bilgir O, Bilgir F, CalanM,CalanOG,Yuksel. Comparison of pre-and post-levothyroxine high-sensitivity $\mathrm{C}$-reactive protein and fetuin-A levels in subclinical hypothyroidism. Clinics(Sao Paulo) 2015;70(2):97-101.

11. Warusevitane A, Karunatilake D, Sim J, Smith C, Roffe C. Early diagnosis of pneumonia in severe stroke: clinical features and the diagnostic role of C-reactive protein. PloS One. 2016;11(3):e0150269.

12. Chalmers S, Khawaja A, Wieruszewski PM, Gajic O, Odeyemi Y. Diagnosis and treatment of acute pulmonary inflammation in critically ill patients: the role of inflammatory biomarkers. World J Crit Care Med. 2019;8(5):59-71.

13. Matsumoto H, Kasai T, Sato A, Ishiwata S, Yatsu S, Shitara J, et al. Association between C-reactive protein levels at hospital admission and long-term mortality in patients with acute decompensated heart failure. Heart Vessels 2019;34(12):1961-1968.

14. Giovanni Ponti, Monia Maccaferri, CristelRuini, Aldo Tomasiand TomrisOzben.Biomarkers associated with COVID-19 disease progression.Crit Rev Clin Lab Sci. 2020;57(6):389-399.

15. Cheng B, Hu J, Zuo X, Chen J, Li X, Chen Y, et al. Predictors of progression from moderate to severe coronavirus disease 2019: a retrospective cohort. Clin Microbiol Infect. 2020;26(10):14001405.

16. Li Q, Ding X, Xia G, Chen H, Chen F, sGeng Z. Eosinopenia and elevated C-reactive protein facilitate triage of COVID-19 patients in fever clinic: a retrospective case-control study. E Clin Med. 2020;23:100375.

17. Chen W, Kenneth I, Zheng S, Liu S, Yan Z, Xu C, et al.Plasma CRP level is positively associated with the severity of COVID-19. Ann Clin Microbiol Antimicrob. 2020; 19:18.

18. Connelly KG, Moss M, Parsons PE, Moore EE, Moore FA, Giclas PC, et al. Serum ferritin as a predictor of the acute respiratory distress syndrome. Am J Respir Crit Care Med. 1997;155:21-25.

19. Shen L,Li S, Zhu Y, Zhao J, Tang X, Li H, et al.Clinical and laboratory derived parameters of 119 hospitalized patients with coronavirus disease 2019 in Xiangyang, Hubei Province. China. J Infect. 2020;81(1):147-178. 